\title{
Crystalline ground state in chiral Gross-Neveu and Cooper pair models at finite densities
}

\author{
Katsumi Ohwat \\ Department of Physics, Tokyo Metropolitan University, \\ 1-1 Minami-Osawa, Hachioji, Tokyo, 192-0397, Japan
}

(November 4, 2018)

\begin{abstract}
We study the possibility of spatially non-uniform ground state in $(1+1)$ dimensional models with quartic fermi interactions at finite fermion densities by introducing chemical potential $\mu$. We examine the chiral Gross-Neveu model and the Cooper pair model as toy models of the chiral symmetry breaking and the difermion pair condensates which are presumed to exist in QCD. We confirm in the chiral Gross-Neveu model that the ground state has a crystalline structure in which the chiral condensate oscillates in space with wave number $2 \mu$. Whereas in the Cooper pair model we find that the vacuum structure is spatially uniform. Some discussions are given to explain this difference.
\end{abstract}

PACS numbers: 11.30.Qc, 11.10.Kk, 11.15.Pg

*k_ohwa@comp.metro-u.ac.jp 


\section{INTRODUCTION}

Illuminating the vacuum structure of QCD at high baryonic densities, which should be useful to understand physics of compact stars and heavy ion collisions, remains challenge for particle and nuclear theorists. One of the most interesting in the related subjects is the color superconductivity of quarks [1, 2, 3]. Quark Cooper pairs condense due to the BCS instability of Fermi surface at high densities and low temperatures, while chiral condensates is dynamically generated by particle-antiparticle pairing at low temperatures and densities. Since quarks have a color component of the $S U(3)$ gauge group, the ground state of QCD is a superconductor with spontaneous breaking of color symmetry as a result of diquark condensates.

In this paper, we address the question of spatial variations of fermionic condensates, the problem first investigated by Deryagin, Grigoriev and Rubakov (DGR) in QCD with infinite number of colors $N_{c}$ [ [ W coupling) and by using the variational method, DGR discovered a previously unsuspected (in the context of QCD) instability of the Fermi surface. Namely, they found that an instability exists in the large $N_{c}$ QCD at finite densities which triggers condensate of particle-hole pairs (Overhauser effect) having almost the same momentum $\boldsymbol{p}$ with modulus $|\boldsymbol{p}|=\mu$, where $\mu$ is the chemical potential. They showed that the ground state of the theory is spatially nonuniform, and has a periodically varying chiral condensate with wave number $2 \mu$.

Following DGR, analyses which utilize either renormalization group analysis [5] or Wilsonian effective action formalism [6] as well as the Nambu-Gorkov formalism [7] were attempted to examine which effect, the Overhauser or the BCS effects wins at large, at finite $N_{c}$. It was shown in these analyses that the BCS effect overtakes the Overhauser effect in weak coupling regime, while Overhauser wins against BCS in strong coupling regime. More recently, the possibility of crystalline ground state was investigated in the context of color superconductivity [8,9, 10]. The underlying mechanism which leads to the crystalline structure is a mismatch in the fermi surface of up and down quarks, and is apparently quite different from one for the periodic chiral condensate discussed by DGR.

To have better understanding of the relationship between these related but different phenomena, it is desirable to have a theoretical laboratory to investigate the underlying mechanism of these different phenomena. In this paper, we examine two simple $(1+1)$ dimensional models with quartic fermi interactions which would serve for this purpose. We examine the chiral Gross-Neveu model [11] and the Cooper pair model proposed by Chodos, Minakata and Cooper [12].

The chiral Gross-Neveu model [11] is a natural choice as the two-dimensional model which displays dynamical breaking of continuous chiral symmetry. It is also well known that the Gross-Neveu model at infinite number of flavor has a number of similar properties with QCD; the renormalizability, the asymptotic freedom, and the similar phase structure on $\mu-T$ plane with those suspected for the two-flavor QCD.

The Cooper pair model [12] is a toy model for "color superconductivity" in QCD but without color degrees of freedom. It nevertheless serves as a renormalizable asymptotically free model field theory which admits Lorentz scalar fermion pair condensate. The model possesses $O(N)$ flavor symmetry and $U(1)$ symmetry of fermion number, and the authors of Ref. 12] have shown that at the large- $N$ limit the $U(1)$ symmetry is dynamically broken by 
the Lorentz singlet di-fermion condensate. It was also shown that the model, when coupled with the Gross-Neveu model, has a very similar phase structure with that of QCD with two flavor of quarks [13].

Strictly speaking, continuous symmetry such as the $U(1)$ symmetries of chirality or fermion number cannot be violated by the Coleman-Mermin-Wagner theorem [14] in $(1+1)$ dimensions. However, it is well understood by now in what sense the theorem is "invalidated" in the large- $N$ limit. As Witten argued [15], the correlation functions of order parameters have power law behavior at large distances, and the power is inversely proportional to $N$. Therefore, if we take the limit $N \rightarrow \infty$ first and then send spatial distance between two order parameters infinity to use the cluster property, we formally obtain nonvanishing vacuum expectation value of the order parameter. The procedure seems to give a consistent field theory, which is usuable as a theoretical laboratory for investigating various properties of condensate phenomena.

We develop a systematic method for investigating the possibilities of spatial varing condensates, and then apply the formalism to discuss the chiral Gross-Neveu model and the Cooper pair model in a unified way. In a recent paper, Schön and Thies [16 treated the chiral Gross-Neveu model and concluded that the model has chiral wave condensates with a wave number $2 \mu$. Our formalism differs from theirs on several respects, in particular on how to regulate ultraviolet divergence. We will reexamine the chiral Gross-Neveu model in the light of our formalism and confirm their results. On the other hand, We obtain a spatially uniform fermion pair condensate as the stable ground state of the Cooper pair model, in agreement with [12].

In Sec. II] we introduce the quasi-particle picture and the Hamiltonian is diagonalized in terms of the quasi-particles. In Sec. III the effective potential is derived by taking the expectation values of the Hamiltonian with respect to properly defined variational vacua and we search for a vacuum solution. In Sec. IV we relate the quasi-particles to the free particles through the Bogoliubov-Valatin transformation, which leads us to the fact that the spatial variations of the chiral condensate yield due to the nonvanishing momentum of particle-hole/antiparticle pairs. We also discuss validity of the ultraviolet regularization by considering the fermion number of vacuum. In Sec. $\mathrm{V}$ the similar discussion to the chiral Gross-Neveu model is applied to the Cooper pair model. Sec. V1 is devoted to conclusions. In Appendix we discuss the ultraviolet regularization scheme in detail.

\section{THE HAMILTONIAN OF CHIRAL GROSS-NEVEU MODEL}

First of all, we will focus our attention on the chiral Gross-Neveu model in which the Lagrangian is given by

$$
\mathcal{L}=\bar{\psi}_{i}\left(i \not \partial+\gamma^{0} \mu\right) \psi_{i}+\frac{1}{2} g^{2}\left|\bar{\psi}_{i}\left(1+\gamma^{5}\right) \psi_{i}\right|^{2} .
$$

Here, $\psi_{i}$ is a two-component spinor with a flavor index of $S U(N)$. Repeated flavor indices in Eq. (1) are meant to be summed. To solve the model, $N$ is sent to an infinite, keeping $\lambda_{0}=g^{2} N$ fixed. We also introduce a chemical potential $\mu$ which is a constant source for fermion number in order to take account of the finite fermion density of the system. The 
Lagrangian has a $U(1)$ chiral symmetry, which is dynamically broken at zero temperature and $\mu=0$ [11]. Under the assumption of spacially uniform ground state the symmetry is shown to be restored at finite temperature and/or chemical potential [17]. A recent work that relaxes the assumption of spatial uniformity to accomodate a periodically varing one [16] has shown that a chiral crystalline state with $\left\langle\bar{\psi}_{i}\left(1+\gamma^{5}\right) \psi_{i}\right\rangle \propto e^{2 i \mu x}$ has lower energy than uniform chiral symmetry breaking or unbroken state. Using this model, we will first develop a method for investigating possibilities of spatially varing condensates, which can also be applied to the Cooper pair model.

Our strategy is to use the auxiliary field method. Following standard techniques [11] we can add the term involving complex, auxiliary field $\sigma(x)$ without affecting on the dynamics of the theory:

$$
\mathcal{L} \rightarrow \mathcal{L}-\frac{1}{2 g^{2}}\left|\sigma+g^{2} \bar{\psi}_{i}\left(1+\gamma^{5}\right) \psi_{i}\right|^{2} .
$$

An effective action of the model is obtained by doing the functional integral with respect to both auxiliary and fermion fields. In the large- $N$ limit, however, $\sigma(x)$ can be regarded as a classical, background field for fermions because it is determined by the method of stationary phase. Instead of integrating out the fermion fields in the effective action, we diagonalize the fermionic Hamiltonian in the presence of the background field $\sigma(x)$ in terms of the creation and annihilation operators of quasi-particles. The free energy is then obtained as expectation values of the Hamiltonian with respect to variational ground states for each $\sigma$. At this moment, since a $U(1)$ chiral transformation including the auxiliary field is indicated by $\psi_{i} \rightarrow e^{i \alpha \gamma^{5}} \psi_{i}$ and $\sigma \rightarrow e^{2 i \alpha} \sigma$ ( $\alpha$ is an arbitrary constant), non-vanishing classical field $\sigma$ implies spontaneous symmetry breaking of the $U(1)$ chiral symmetry.

To analyze the chiral Gross-Neveu model and the Cooper pair model in parallel, it is convenient to introduce the charge conjugation field defined by

$$
\psi_{i}^{c}(x)=C \bar{\psi}_{i}^{T}(x),
$$

where $C$ is a $2 \times 2$ matrix with the properties (see, for example, Bjorken and Drell [18])

$$
C^{-1} \gamma^{\mu} C=-\gamma^{\mu T}, \quad C=-C^{T} .
$$

In our convention of Dirac matrices, $\gamma^{0}=\sigma_{1}, \gamma^{1}=-i \sigma_{2}$ and $\gamma^{5}=\sigma_{3}$ ( $\sigma^{\prime}$ s are Pauli matrices), $C=-\gamma^{1}$ and charge conjugation field is described as

$$
\psi_{i}^{c}(x)=\gamma^{5} \psi_{i}^{*}(x) .
$$

By using the integral identity

$$
\frac{1}{2} \int d^{2} x \bar{\psi}_{i}\left(i \not \supset+\gamma^{0} \mu\right) \psi_{i}=\frac{1}{2} \int d^{2} x \bar{\psi}_{i}^{c}\left(i \not \supset-\gamma^{0} \mu\right) \psi_{i}^{c},
$$

the fermionic Hamiltonian density corresponding to the Lagrangian of the chiral Gross-Neveu model can be written as 


$$
\begin{aligned}
\mathcal{H} & =\frac{\partial \mathcal{L}}{\partial \dot{\psi}_{i}} \psi_{i}+\frac{\partial \mathcal{L}}{\partial \dot{\psi}_{i}^{c}} \psi_{i}^{c}-\mathcal{L} \\
& =\frac{|\sigma|^{2}}{2 g^{2}}+\frac{1}{2}\left[{\psi_{i}}^{\dagger}, \psi_{i}^{c \dagger}\right]\left[\begin{array}{cc}
h & 0 \\
0 & h^{c}
\end{array}\right]\left[\begin{array}{c}
\psi_{i} \\
\psi_{i}^{c}
\end{array}\right],
\end{aligned}
$$

where the first quantized Hamiltonian $\hat{H}=\left[\begin{array}{cc}h & 0 \\ 0 & h^{c}\end{array}\right]$ is given by

$$
\begin{aligned}
h & =-i \gamma^{5} \partial_{x}-\mu+\gamma^{0} \operatorname{Re} \sigma-i \gamma^{1} \operatorname{Im} \sigma \\
h^{c} & =-i \gamma^{5} \partial_{x}+\mu+\gamma^{0} \operatorname{Re} \sigma+i \gamma^{1} \operatorname{Im} \sigma
\end{aligned}
$$

Note that the sign of $\operatorname{Im} \sigma$ term flips because the charge conjugation is a kind of chiral conjugation (see, Eq. (3)) and the imaginary part of an auxiliary field $\sigma$ transforms as $\bar{\psi}_{i} \gamma^{5} \psi_{i}$.

Our next task is to identify the eigenstates of Eq. (5) and to expand the fermion field in terms of them. To do this, we assume that $\sigma(x)$ has the following static standing wave form:

$$
\sigma(x)=|\sigma| e^{2 i K x}
$$

where $|\sigma|$ and $K$ are arbitary constants which will be determined later. We solve eigenvalue problem $\hat{H} \Psi=\omega_{n} \Psi$ by Fourier expanding with the chiral phase factor $e^{ \pm i K x \gamma^{5}}$. We obtain a complete, orthonormal set of eigen functions $\Psi=\left[\psi_{n}(x), \psi_{n}^{c}(x)\right]^{T}$. It consists of four types of continuum state labeled as $n=(p, a),(p, b),(-p, \bar{a}),(-p, \bar{b})$ with the following eigenvalus ( $p$ is the Fourier mode and we call this type of eigenstates "branch", e.g., $a$-branch):

$$
\begin{array}{lll}
\omega_{p a}=\epsilon_{p}-\mu_{*} & : \psi_{p a}(x)=e^{i\left(p+\gamma^{5} K\right) x} u_{p} & , \psi_{p a}^{c}(x)=0, \\
\omega_{p b}=\epsilon_{p}+\mu_{*} & : \psi_{p b}(x)=0 & , \psi_{p b}^{c}(x)=e^{i\left(p-\gamma^{5} K\right) x} u_{p}, \\
\omega_{-p \bar{a}}=-\epsilon_{p}+\mu_{*}: \psi_{-p \bar{a}}(x)=0 & , \psi_{-p \bar{a}}^{c}(x)=e^{i\left(p-\gamma^{5} K\right) x} u_{-p}^{c}, \\
\omega_{-p \bar{b}}=-\epsilon_{p}-\mu_{*}: \psi_{-p \bar{b}}(x)=e^{i\left(p+\gamma^{5} K\right) x} u_{-p}^{c} & , \psi_{-p \bar{b}}^{c}(x)=0 .
\end{array}
$$

Here, $\epsilon_{p}=\sqrt{p^{2}+|\sigma|^{2}}$ and $\mu_{*}=\mu-K$. The two component spinors in Eqs. (\$) are

$$
\begin{aligned}
u_{p} & =\left[\begin{array}{c}
\cos \frac{1}{2} \theta_{p} \\
\sin \frac{1}{2} \theta_{p}
\end{array}\right]=\frac{1}{\sqrt{2 \epsilon_{p}}}\left[\begin{array}{c}
\sqrt{\epsilon_{p}+p} \\
\sqrt{\epsilon_{p}-p}
\end{array}\right], \\
u_{-p}^{c}=\gamma^{5} u_{-p}^{*} & =\left[\begin{array}{c}
\sin \frac{1}{2} \theta_{p} \\
-\cos \frac{1}{2} \theta_{p}
\end{array}\right]=\frac{1}{\sqrt{2 \epsilon_{p}}}\left[\begin{array}{c}
\sqrt{\epsilon_{p}-p} \\
-\sqrt{\epsilon_{p}+p}
\end{array}\right] .
\end{aligned}
$$

Note that the wave functions are no longer momentum eigenstates in the presense of $K$ because of the chiral phase factor. While $\omega$ 's may be regarded as the energies of a fermion or an antifermion with momentum $p$, mass $|\sigma|$ and chemical potential $\mu_{*}$, they must be interpreted merely as quasi-particle dispersion relations (especially, $p$ is not a momentum of particles).

The fermion field can be expanded in terms of eigenstates (8). The coefficients of four branches of eigenstates are not independent with each other, but $a(b)$ - and $\bar{a}(\bar{b})$-branches 
must be hermitian conjugate with each other to assure that $\psi_{i}^{c}=\gamma^{5} \psi_{i}^{*}$. The second quantized fermion field is then given by

$$
\left[\begin{array}{c}
\psi_{i}(x) \\
\psi_{i}^{c}(x)
\end{array}\right]=\int \frac{d p}{2 \pi}\left[\begin{array}{l}
e^{i\left(p+\gamma^{5} K\right) x}\left(u_{p} a_{p i}+u_{-p}^{c} b_{-p i}^{\dagger}\right) \\
e^{i\left(p-\gamma^{5} K\right) x}\left(u_{-p}^{c} a_{-p i}^{\dagger}+u_{p} b_{p i}\right)
\end{array}\right] .
$$

Here, $a_{p i}, b_{p i}$ and their hermitian conjugates are creation and annihilation operators of quasiparticles. To hold the canonical anticommutation relations, $\left\{\psi_{i}(x), \psi_{j}^{\dagger}(y)\right\}=\delta_{i j} \delta(x-y)$,

they must obey anticommutation relations; $\left\{a_{p i}, a_{q j}^{\dagger}\right\}=\left\{b_{p i}, b_{q j}^{\dagger}\right\}=2 \pi \delta(p-q) \delta_{i j}$, and all other anticommutators vanish.

Using the decomposition into Fourier space, the field operators can be expressed in terms of quasi-particle operators, $a$ 's and $b$ 's. For example, Hamiltonian and fermion number operator become

$$
\begin{aligned}
H & =\int d x \mathcal{H} \\
& =N L \frac{|\sigma|^{2}}{2 \lambda_{0}}+\frac{1}{2} \int \frac{d p}{2 \pi}\left(\omega_{p a} a_{p i}^{\dagger} a_{p i}+\omega_{p b} b_{p i}^{\dagger} b_{p i}+\omega_{-p \bar{a}} a_{-p i} a_{-p i}^{\dagger}+\omega_{-p \bar{b}} b_{-p i} b_{-p i}^{\dagger}\right), \\
J^{0} & =\frac{1}{2} \int d x\left[\psi_{i}^{\dagger} \psi_{i}-\psi_{i}^{c \dagger} \psi_{i}^{c}\right] \\
& =\frac{1}{2} \int \frac{d p}{2 \pi}\left(a_{p i}^{\dagger} a_{p i}-b_{p i}^{\dagger} b_{p i}-a_{-p i} a_{-p i}^{\dagger}+b_{-p i} b_{-p i}^{\dagger}\right),
\end{aligned}
$$

where $L=\int d x$ is the spatial volume. It is found that $a_{p i}^{\dagger}$ is the annihilation operator of the $\bar{a}$-branch simultaneously with the creation operator of the $a$-branch. It should be also noticed that the quasi-particle operators diagonalize the fermion number operator differently from the Cooper pair model. This becomes the crucial point to distinguish between the two models, as one can find below.

\section{EFFECTIVE POTENTIAL AND VACUUM STRUCTURE FOR CHIRAL GROSS-NEVEU MODEL}

In this section we want to construct and analyze the effective potential for the chiral Gross-Neveu model. We begin by calculating expectation values of the Hamiltonian with respect to variational ground state which is defined by filling the negative energy states;

$$
\begin{aligned}
& \omega_{p a}=-\omega_{p \bar{a}}>0: a_{p i}|\sigma\rangle=0, \omega_{p b}=-\omega_{p \bar{b}}>0: b_{p i}|\sigma\rangle=0 \\
& \omega_{p a}=-\omega_{p \bar{a}}<0: a_{p i}^{\dagger}|\sigma\rangle=0, \omega_{p b}=-\omega_{p \bar{b}}<0: b_{p i}^{\dagger}|\sigma\rangle=0
\end{aligned} .
$$

Evaluating the expectation value of the Hamiltonian with respect to $|\sigma\rangle$, the effective potential becomes (for brevity, we denote $\langle\mathcal{O}\rangle=\langle\sigma|\mathcal{O}| \sigma\rangle$ )

$$
\begin{aligned}
\mathcal{V}(|\sigma|, K) & =\frac{\langle H\rangle}{N L} \\
& =\frac{|\sigma|^{2}}{2 \lambda_{0}}+\frac{1}{2} \int \frac{d p}{2 \pi} \omega_{-p \bar{a}}+\frac{1}{2} \int \frac{d p}{2 \pi} \omega_{-p \bar{b}}+\int_{\omega_{p a}<0} \frac{d p}{2 \pi} \omega_{p a}+\int_{\omega_{p b}<0} \frac{d p}{2 \pi} \omega_{p b},
\end{aligned}
$$


where the region of integration in the second and the third terms in the last line consists of whole range of $p$. The last two integrals come from "no-pairing" regions in which the modes does not give any contribution to dynamical generation of chiral condensate (see, e.g, Eq. (21)). Under the condition for absence of no-paring regions, $\mu_{*}^{2}<|\sigma|^{2}$, there is no effect from free fermions and these integrals vanish.

Eq. (14) contains several divergences at ultraviolet regions; for example, if we introduce the naive Fourier mode cutoff (as we took care before, $p$ is not a momentum of quasi-particle), $|p|<\Lambda / 2$, then the divergent terms come from first two integrals in Eq. (14):

$$
\begin{aligned}
& \int_{-\Lambda / 2}^{\Lambda / 2} \frac{d p}{2 \pi} \omega_{-p \bar{a}}=-\frac{\Lambda^{2}}{8 \pi}+\frac{\mu_{*}}{2 \pi} \Lambda-\frac{|\sigma|^{2}}{4 \pi} \ln \frac{\Lambda^{2}}{|\sigma|^{2}}-\frac{|\sigma|^{2}}{2 \pi}+\mathcal{O}(1 / \Lambda) \\
& \int_{-\Lambda / 2}^{\Lambda / 2} \frac{d p}{2 \pi} \omega_{-p \bar{b}}=-\frac{\Lambda^{2}}{8 \pi}-\frac{\mu_{*}}{2 \pi} \Lambda-\frac{|\sigma|^{2}}{4 \pi} \ln \frac{\Lambda^{2}}{|\sigma|^{2}}-\frac{|\sigma|^{2}}{2 \pi}+\mathcal{O}(1 / \Lambda)
\end{aligned}
$$

The quadratic and linear divergences depend on the cutoff scheme, although the logarithmic divergence does not. These divergences therefore lead to nontrivial issue for the ultraviolet regularization. We will show at the next section that the naive Fourier mode cutoff has problems with the number of fermions floating in the vacuum. We employ a different cutoff scheme which is free from the trouble. As will be explained somewhat in detail in Appendix, it involves the branch dependent cutoff prescription:

$$
a(\bar{a}) \text {-branch : }\left[-\frac{\Lambda}{2}+K, \frac{\Lambda}{2}-K\right], \quad b(\bar{b}) \text {-branch : }\left[-\frac{\Lambda}{2}-K, \frac{\Lambda}{2}+K\right] .
$$

The reader may wonder why one should take such branch-dependent shift on the ultraviolet cutoff. In fact, it is shown in Refs. [19] that the similar shift resulting from a phase shift in the normal modes of fluctuations around the soliton gives correct quantum corrections to the kink mass in the two-dimentional $\phi^{4}$-theory.

By using the cutoff prescription (16), first two integrals in the effective potential (14) become

$$
\begin{aligned}
& \int_{-\Lambda / 2+K}^{\Lambda / 2-K} \frac{d p}{2 \pi} \omega_{-p \bar{a}}=-\frac{\Lambda^{2}}{8 \pi}+\frac{\mu}{2 \pi} \Lambda-\frac{|\sigma|^{2}}{4 \pi} \ln \frac{\Lambda^{2}}{|\sigma|^{2}}-\frac{|\sigma|^{2}}{2 \pi}+\frac{\mu_{*}^{2}}{2 \pi}-\frac{\mu^{2}}{2 \pi}+\mathcal{O}(1 / \Lambda) \\
& \int_{-\Lambda / 2-K}^{\Lambda / 2+K} \frac{d p}{2 \pi} \omega_{-p \bar{b}}=-\frac{\Lambda^{2}}{8 \pi}-\frac{\mu}{2 \pi} \Lambda-\frac{|\sigma|^{2}}{4 \pi} \ln \frac{\Lambda^{2}}{|\sigma|^{2}}-\frac{|\sigma|^{2}}{2 \pi}+\frac{\mu_{*}^{2}}{2 \pi}-\frac{\mu^{2}}{2 \pi}+\mathcal{O}(1 / \Lambda)
\end{aligned}
$$

One notices by comparing Eqs. (17) to (15) that additional finite contributions arise from the lineary or quadraticaly divergent terms due to the shifts in cutoff. The quadratic divergence in Eq. (17) is an uninteresting constant which can be subtracted off and the linear divergences are exactly cancelled between two integrals. The remaining logarithmic divergence in Eqs. (17) can be removed, as usual, by the renormalization of the coupling constant. Since they contain only $|\sigma|$ dependence, it is enough to carry it out in the case of $K=\mu=0$. Therefore, we renormalize by demanding that the renormalized coupling constant $\lambda$ satisfies 


$$
\left.\frac{\partial^{2} \mathcal{V}}{\partial|\sigma|^{2}}\right|_{\substack{|\sigma|=m_{0} \\ K=\mu=0}}=\frac{1}{\lambda}
$$

Here $|\sigma|=m_{0}$ designates an arbitary renormalization point on which the coupling constant depends. Using this conditions to solve for $\lambda_{0}$ in terms of $\lambda$ yields the renormalized form of the effective potential:

$$
\begin{aligned}
\mu_{*}^{2}<|\sigma|^{2}: \mathcal{V}=\frac{|\sigma|^{2}}{4 \pi}\left[\frac{2 \pi}{\lambda}-3+\ln \frac{|\sigma|^{2}}{m_{0}^{2}}\right]+\frac{\mu_{*}^{2}}{2 \pi}-\frac{\mu^{2}}{2 \pi} \\
\mu_{*}^{2}>|\sigma|^{2}: \mathcal{V}=\frac{|\sigma|^{2}}{4 \pi}\left\{\frac{2 \pi}{\lambda}-3+\ln \frac{\mu_{*}^{2}}{m_{0}^{2}}\left[1+\sqrt{1-\frac{|\sigma|^{2}}{\mu_{*}^{2}}}\right]^{2}\right\} \\
+\frac{\mu_{*}^{2}}{2 \pi}\left[1-\sqrt{1-\frac{|\sigma|^{2}}{\mu_{*}^{2}}}\right]-\frac{\mu^{2}}{2 \pi} .
\end{aligned}
$$

When $K=0$, Eqs. (19) turns into the well-known effective potential assuming the homogeneity of chiral condensates [17]. If we set $K=\mu$, the effective potential is reduced to one in Ref. [16].

It is easy to show that $|\sigma|=m_{0} e^{1-\pi / \lambda}, \mu_{*}=\mu-K=0$ is the solution of the gap equations derived from the effective potential, $\frac{\partial \mathcal{V}}{\partial|\sigma|}=\frac{\partial \mathcal{V}}{\partial K}=0$. It give the minmum value of effective potential as one can see in Fig. 1. Therefore, the vacuum structure is characterized by

$$
\sigma(x)=\left(m_{0} e^{1-\pi / \lambda}\right) e^{2 i \mu x} .
$$

Using the field decomposition (10), one can evaluate the chiral condensate of vacuum explicitly,

$$
\begin{aligned}
\left\langle\bar{\psi}_{i}\left(1+\gamma^{5}\right) \psi_{i}\right\rangle & =-N|\sigma| e^{2 i K x} \frac{1}{2 \pi}\left[\int \frac{d p}{\epsilon_{p}}-\int_{\omega_{p a}<0} \frac{d p}{\epsilon_{p}}-\int_{\omega_{p b}<0} \frac{d p}{\epsilon_{p}}\right] \\
& =-N \sigma(x) / \lambda_{0},
\end{aligned}
$$

where the vacuum value of $\sigma(x)$, Eq. (20), is used in the second line of the equation. The equation guarantees to hold the equation of motion for $\sigma(x)$ derived from the Lagrangian (2). Consequently, we have reproduced the result in Ref. [16 which the phase standing chiral density wave with a wave number $2 \mu$ is stable as compared to uniformally chiral symmetry broken phase or the symmetric phase. Moreover, we have demonstrated that the condensate of the wave number $2 \mu$ arises as the unique minimum of the effective potential. We present arguments to clarify the reasons why the wave number is $2 \mu$ at the next section.

\section{RELATION TO THE OVERHAUSER EFFECT}

To understand why the chiral condensate oscillates in space with wave number $2 \mu$, we start by considering that the field expansion, Eq. (10), has a connection with an interactionfree description through the Bogoliubov-Valatin transformation. For brevity, we drop the flavor indices and set $L$ to unity. Setting $|\sigma|=K=0$ in Eq. (10), the free field expansion is 


$$
\psi(x)=\int \frac{d q}{2 \pi} e^{i q x}\left[\begin{array}{c}
a_{q}^{(0)} \Theta(q)+b_{-q}^{(0) \dagger} \Theta(-q) \\
a_{q}^{(0)} \Theta(-q)-b_{-q}^{(0) \dagger} \Theta(q)
\end{array}\right],
$$

where the subscript (0) implies the operators corresponding to free massless fermion and antifermion, and $\Theta$ denotes the Heaviside step function. By equating Eq. (22) to Eq. (10), the Bogoliubov-Valatin transformation reads

$$
\begin{array}{r}
K<p:\left\{\begin{array}{l}
a_{p}=a_{p+K}^{(0)} \cos \frac{1}{2} \theta_{p}-b_{-p+K}^{(0) \dagger} \sin \frac{1}{2} \theta_{p}, \\
b_{p}=b_{p-K}^{(0)} \cos \frac{1}{2} \theta_{p}-a_{-p-K}^{(0) \dagger} \sin \frac{1}{2} \theta_{p},
\end{array}\right. \\
-K<p<K:\left\{\begin{array}{l}
a_{p}=a_{p+K}^{(0)} \cos \frac{1}{2} \theta_{p}+a_{p-K}^{(0)} \sin \frac{1}{2} \theta_{p}, \\
b_{p}=a_{-p+K}^{(0) \dagger} \cos \frac{1}{2} \theta_{p}-a_{-p-K}^{(0) \dagger} \sin \frac{1}{2} \theta_{p},
\end{array}\right. \\
p<-K:\left\{\begin{array}{l}
a_{p}=b_{-p-K}^{(0) \dagger} \cos \frac{1}{2} \theta_{p}+a_{p-K}^{(0)} \sin \frac{1}{2} \theta_{p}, \\
b_{p}=a_{-p+K}^{(0) \dagger} \cos \frac{1}{2} \theta_{p}+b_{p+K}^{(0)} \sin \frac{1}{2} \theta_{p} .
\end{array}\right.
\end{array}
$$

We can then relate the variational ground state $|\sigma\rangle$ to the normal state $|0\rangle$. The latter is annihilated by $a^{(0)}\left(a^{(0) \dagger}\right)$ and $b^{(0)}$ above (below) the Fermi surface:

$$
\begin{aligned}
& |q|>\mu: a_{q}^{(0)}|0\rangle=b_{q}^{(0)}|0\rangle=0, \\
& |q|<\mu: a_{q}^{(0) \dagger}|0\rangle=b_{q}^{(0)}|0\rangle=0,
\end{aligned}
$$

(We implicitly assume that $\mu>0$ such that the fermionic Fermi surface is formed). For the case of $K=\mu$ in particular, one is able to show that $|\sigma\rangle$ can be written in a form of the BCS state,

$$
|\sigma\rangle=\prod_{p<-\mu} A_{p}^{-\dagger} \prod_{-\mu<p<0} B_{p}^{-\dagger} \prod_{0<p<\mu} B_{p}^{+\dagger} \prod_{\mu<p} A_{p}^{+\dagger}|0\rangle,
$$

where

$$
\begin{aligned}
& A_{p}^{+\dagger}=\cos \frac{1}{2} \theta_{p}+\sin \frac{1}{2} \theta_{p} a_{p+\mu}^{(0) \dagger} b_{-p+\mu}^{(0) \dagger}, \\
& B_{p}^{+\dagger}=\cos \frac{1}{2} \theta_{p}+\sin \frac{1}{2} \theta_{p} a_{p-\mu}^{(0)} a_{p+\mu}^{(0) \dagger}, \\
& B_{p}^{-\dagger}=\sin \frac{1}{2} \theta_{p}+\cos \frac{1}{2} \theta_{p} a_{p+\mu}^{(0)} a_{p-\mu}^{(0) \dagger} . \\
& A_{p}^{-\dagger}=\sin \frac{1}{2} \theta_{p}+\cos \frac{1}{2} \theta_{p} b_{-p-\mu}^{(0) \dagger} a_{p-\mu}^{(0) \dagger},
\end{aligned}
$$

It can be easily verified that $|\sigma\rangle$ in Eq. (25) satisfies the definition of it, Eq. (13), by using Eqs. (23) and (24). In the region of $p>\mu,|\sigma\rangle$ comprises with the particle-antiparticle pairing as in the usual chiral condensate vacua but with pair momentum $2 \mu$ in our case. On the other hand, in the region of $0<p<\mu,|\sigma\rangle$ comprises the particle-hole pairing with the momentum $(p+\mu)$ and $-(p-\mu)$, respectively. The manner of pairing is depicted in Fig. 2. This figure tells us that either the particle-hole or the particle-antiparticle pairing across the Fermi surface replaces particle-antiparticle pairing across the Dirac sea that generates homogeneous chiral condensates at $\mu=0$. Exciting the pair of a particle with momentum $\mu$ and a hole with momentum $\mu(p=0)$ costs zero energy. Since the pairing wave function $\tan \frac{1}{2} \theta_{p}$ at $p>0$ has the peak at $p=0$, most likely, the zero energy pairing is generated when the interaction is truned on. This is the Overhauser instability first proposed for spin 
density wave of nonrelativistic electron system [20], and recently revived for chiral density wave in QCD [4,5,66,7]. Because of effects of this instability, the vacuum must have the chiral crystalline structure with a period $\pi / \mu$.

Eq. (25) tells us an another important result. Since $|0\rangle$ has nonzero fermion number owing to the existence of the Fermi sea and the pairing preserves fermion number, $|\sigma\rangle$ must also have nonzero fermion number. The expectation value of a fermion number operator becomes

$$
\begin{aligned}
\left\langle J^{0}\right\rangle & =-\frac{1}{2} \int_{-\Lambda / 2+K}^{\Lambda / 2-K} \frac{d p}{2 \pi} N L+\frac{1}{2} \int_{-\Lambda / 2-K}^{\Lambda / 2+K} \frac{d p}{2 \pi} N L+\int_{\omega_{p a}<0} \frac{d p}{2 \pi} N L-\int_{\omega_{p b}<0} \frac{d p}{2 \pi} N L \\
& = \begin{cases}N L \frac{K}{\pi} \quad \text { for } \mu_{*}^{2}<|\sigma|^{2} \\
N L\left(\frac{K}{\pi}+\frac{\mu_{*}}{\pi} \sqrt{1-\frac{|\sigma|^{2}}{\mu_{*}^{2}}}\right) & \text { for } \mu_{*}^{2}>|\sigma|^{2}\end{cases}
\end{aligned}
$$

by our cutoff scheme (16). At $\mu_{*}=0$, or equivalently $K=\mu$, it yields the correct fermion number of the state $|\sigma\rangle,\left\langle J^{0}\right\rangle=N L \mu / \pi$. We emphasize that the consistent result comes out because of the cutoff procedure (16) we employ. Vanishing fermion number would result if the naive Fourier mode cutoff were used.

We comment out about the relation of Eq. (27) to early works relating on the zero chemical potential system, finally. In the chiral sigma model in which $\sigma(x)$ turns into the true backgroun field, the authors of Ref. [21] has shown that the vacuum expectation value

of fermion number density is $\left\langle j^{0}\right\rangle=-\frac{\partial_{x} \varphi}{2 \pi}$, where $\sigma(x)=|\sigma| e^{-i \varphi(x) \gamma^{5}}$, by using the adiabatic method. To compare with our model we set $\varphi(x)=-2 K x$, so that the fermion number density becomes the first case of Eq. (27) expecting the factor $N$ coming from the number of flavor. Since the adiabatic method implies the slowly varing condition $\left(\partial_{x} \varphi \ll|\sigma|\right)$, the secound case of Eq. (27) is excluded from this condition.

\section{THE COOPER PAIR MODEL}

We discuss the Cooper pair model in this section, replacing the interaction term in Eq. (1) with $\frac{1}{2} g^{2}\left|\bar{\psi}{ }_{i}^{c} \psi_{i}\right|^{2}$ :

$$
\mathcal{L}=\bar{\psi}_{i}\left(i \not \partial+\gamma^{0} \mu\right) \psi_{i}+\frac{1}{2} g^{2}\left|\bar{\psi}_{i}^{c} \psi_{i}\right|^{2}
$$

The Lagrangian (28) is the same as the one in Ref. [12], due to our convention of Dirac matrices. The Lagrangian has a $U(1)$ symmetry which implies the conserved fermion number in the model. As was shown in Ref. [12] under the assumption of spatilly homogenuity, a Lorentz scalar Cooper pair condensate is dynamically generated in this model; $\left\langle\bar{\psi}_{i}^{c} \psi_{i}\right\rangle \neq 0$ ( $\psi_{i}^{c}$ transform as $\psi_{i}$ under the Lorentz transformations).

We introduce an auxiliary field $\Delta(x)$ by

$$
\mathcal{L} \rightarrow \mathcal{L}-\frac{1}{2 g^{2}}\left|\Delta+g^{2} \bar{\psi}_{i}^{c} \psi_{i}\right|^{2}
$$


When one thinks about the Cooper pair condensate, it is convenient to regard the charge conjugation of $\psi_{i}$ as independent variable of the theory (see, for example, Refs. [1,22]). By introducing the kinetic term of charge conjugation field by Eq. (四), we obtain the Hamiltonian density in the following form,

$$
\mathcal{H}=\frac{|\Delta|^{2}}{2 g^{2}}+\frac{1}{2}\left[\psi_{i}^{\dagger}, \psi_{i}^{c \dagger}\right]\left[\begin{array}{cc}
-i \gamma^{5} \partial_{x}-\mu & \gamma^{0} \Delta \\
\gamma^{0} \Delta^{*} & -i \gamma^{5} \partial_{x}+\mu
\end{array}\right]\left[\begin{array}{c}
\psi_{i} \\
\psi_{i}^{c}
\end{array}\right]
$$

As in the case of chiral Gross-Neveu model, we impose the standing wave ansatz for the background (auxiliary) field:

$$
\Delta(x)=|\Delta| e^{2 i K x}
$$

$\Delta(x)$ is equal to the Cooper pair condensate $\left\langle\bar{\psi}_{i}^{c} \psi_{i}\right\rangle$ apart from a constant factor, so that nonvanishing $|\Delta|$ indicates the symmetry breaking of fermion number $U(1)$ symmetry.

Eigenvalues of the first quantized Hamiltonian in Eq. (30) consist of the following four branches:

$$
\begin{aligned}
& \omega_{p a}=\epsilon_{p_{+}}+K, \omega_{-p \bar{a}}=-\epsilon_{p_{-}}-K \\
& \omega_{p b}=\epsilon_{p_{-}}-K, \quad \omega_{-p \bar{b}}=-\epsilon_{p_{+}}+K
\end{aligned}
$$

where $\epsilon_{p}=\sqrt{p^{2}+|\Delta|^{2}}$ is a energy of a particle with momentum $p$ and mass $|\Delta|$, and $p_{ \pm}=$ $p \mp \mu$ are momenta relative to the Fermi surface. We can then diagonalize the Hamiltonian, and fermionic field is expanded in terms of the eigenstates with phase factor, $e^{ \pm i K x}$ :

$$
\begin{aligned}
& \psi_{i}(x)=\int \frac{d p}{2 \pi} e^{i(p+K) x}\left[\begin{array}{l}
a_{p i} \cos \frac{1}{2} \theta_{p_{+}}+b_{-p i}^{\dagger} \sin \frac{1}{2} \theta_{p_{+}} \\
b_{p i} \sin \frac{1}{2} \theta_{p_{-}}-a_{-p i}^{\dagger} \cos \frac{1}{2} \theta_{p_{-}}
\end{array}\right], \\
& \psi_{i}^{c}(x)=\int \frac{d p}{2 \pi} e^{i(p-K) x}\left[\begin{array}{l}
b_{p i} \cos \frac{1}{2} \theta_{p_{-}}+a_{-p i}^{\dagger} \sin \frac{1}{2} \theta_{p_{-}} \\
a_{p i} \sin \frac{1}{2} \theta_{p_{+}}-b_{-p i}^{\dagger} \cos \frac{1}{2} \theta_{p_{+}}
\end{array}\right] .
\end{aligned}
$$

The wave functions $\cos \frac{1}{2} \theta_{p}$ and $\sin \frac{1}{2} \theta_{p}$ are defined analogously in Eq. (9). The Hamiltonian is obtained in exactly the same form as in Eq. (11) with the energies of Eq. (32).

Decomposing the fermion number and current operator in the basis of Eq. (33) manifests the difference from the chiral Gross-Neveu model. In the Cooper pair model, fermion current is diagonalized by the quasi-particle operators as the following equation, but fermion number is not;

$$
\begin{aligned}
J^{1} & =\frac{1}{2} \int d x\left[\psi_{i}^{\dagger} \gamma^{5} \psi_{i}-\psi_{i}^{c \dagger} \gamma^{5} \psi_{i}^{c}\right] \\
& =\frac{1}{2} \int \frac{d p}{2 \pi}\left(a_{p i}^{\dagger} a_{p i}-b_{p i}^{\dagger} b_{p i}-a_{-p i} a_{-p i}^{\dagger}+b_{-p i} b_{-p i}^{\dagger}\right) .
\end{aligned}
$$

The cutoff scheme is the same one as the chiral Gross-Neveu model, because the field expansion (33) must reduce to the free theory even if $|\Delta|=\mu=0$ and $K \neq 0$ (see Appendix). Variational ground state $|\Delta\rangle$ is defined by filling the negative energy states in the same way as Eq. (13), and then the effective potential becomes 


$$
\begin{aligned}
\mathcal{V}(|\Delta|, K ; \mu)= & \frac{\langle H\rangle}{N L} \\
=\frac{|\Delta|^{2}}{2 \lambda_{0}}+ & \frac{1}{2} \int_{-\Lambda / 2+K}^{\Lambda / 2-K} \frac{d p}{2 \pi} \omega_{-p \bar{a}}+\frac{1}{2} \int_{-\Lambda / 2-K}^{\Lambda / 2+K} \frac{d p}{2 \pi} \omega_{-p \bar{b}} \\
& +\int_{\omega_{p a}<0} \frac{d p}{2 \pi} \omega_{p a}+\int_{\omega_{p b}<0} \frac{d p}{2 \pi} \omega_{p b} .
\end{aligned}
$$

In (35), $\langle H\rangle$ implies to take expectation value with respect to the state $|\Delta\rangle$. Performing the integrations explicitly and using the renormalization condition (18) (replacing $|\sigma|$ to $|\Delta|$ in the equation), we obtain the renormalized effective potential as

$$
\begin{aligned}
K^{2}<|\Delta|^{2}: \mathcal{V}=\frac{|\Delta|^{2}}{4 \pi}\left[\frac{2 \pi}{\lambda}-3+\ln \frac{|\Delta|^{2}}{m_{0}^{2}}\right]+\frac{K^{2}}{2 \pi}-\frac{\mu^{2}}{2 \pi}, \\
K^{2}>|\Delta|^{2}: \mathcal{V}=\frac{|\Delta|^{2}}{4 \pi}\left\{\frac{2 \pi}{\lambda}-3+\ln \frac{K^{2}}{m_{0}^{2}}\left[1+\sqrt{1-\frac{|\Delta|^{2}}{K^{2}}}\right]^{2}\right\} \\
+\frac{K^{2}}{2 \pi}\left[1-\sqrt{1-\frac{|\Delta|^{2}}{K^{2}}}\right]-\frac{\mu^{2}}{2 \pi} .
\end{aligned}
$$

It is easy to observe that the effective potential is identical with that of the chiral GrossNeveu model, Eq. (19), under the replacement of $\mu_{*}$ by $K$. Then, the vacuum is obtained at $K=0$, and the Cooper pair condensate of vacuum is given by

$$
\Delta(x)=m_{0} e^{1-\pi / \lambda}=-\lambda_{0}\left\langle\bar{\psi}_{i}^{c} \psi_{i}\right\rangle / N
$$

(The last equality is straightforwardly proved, similarly to Eq. (21) in the chiral Gross-Neveu model). Therefore, the ground state with spatially uniform Cooper pair condensate obtained in Ref. [12] gives the true vacuum in this model.

The question we have to ask here is why the Cooper pair condensate is uniform differently from the chiral condensate in the chiral Gross-Neveu model. In order to answer this question, we had to better examine the fermion number and current of the variational ground state $|\Delta\rangle$. After some calculations, they turn out to be

$$
\begin{aligned}
& \left\langle J^{0}\right\rangle=N L \frac{\mu}{\pi}, \\
& \left\langle J^{1}\right\rangle=\left\{\begin{array}{cl}
N L \frac{K}{\pi} & \text { for } K^{2}<|\Delta|^{2} \\
N L\left(\frac{K}{\pi}-\frac{K}{\pi} \sqrt{1-\frac{|\Delta|^{2}}{K^{2}}}\right) & \text { for } K^{2}>|\Delta|^{2}
\end{array}\right.
\end{aligned}
$$

The fermion number is independent of the parameter indicating the spatial varidity $K$ in constrast to the chiral Gross-Neveu model. The spatial homogeneity can be explicitly broken in the chiral Gross-Neveu model because the fermion number is an order parameter for this symmetry breaking and it has a source, namely chemical potential. In the Cooper pair model 
this symmetry breakdown, if it occurs, must be caused spontaneously because the fermion number is no longer the order parameter. Then, the spontaneous symmetry breaking of the spatial homogeneity does not occur in our models.

\section{CONCLUSIONS}

In this paper we have analyzed the vacuum structure of $(1+1)$-dimensional models with quartic fermi interactions at finite densities, with particular emphasis on the possibility of spatially nonuniform ground state. Our treatment is restricted into the case of large- $N$ limit and of zero temperature. This is the unique place where we can have nonvanishing order parameter in $1+1$ dimensions in the sense explained in Introduction.

We first examined the chiral Gross-Neveu model. We have constructed the effective potential by allowing the chiral condensate to vary periodically in space. The quasi-particle basis diagonalizing the fermionic Hamiltonian of the theory was obtained by the Fourier expansion after performing the chiral rotation. We have constructed an effective potential and have shown that the vacuum has a crystalline structure in which the chiral condensate oscillates with the wave number $2 \mu$. This is consistent with the result obtained by Schön and Thies [16].

In the course of constructing the effective potential, we have found that the branchdependent ultraviolet cutoff procedure is required to derive the correct effective potential. In the cutoff scheme, the shifts from the explicit cutoff parameter play important role for the purpose of providing the system with the correct fermion number. While the branchdependent ultraviolet cutoff procedure may look ugly, it is likely that it is required to preserve the fermion number in the system by cancelling the spectral flow induced by chiral rotation performed to obtain the basis used in our treatment.

We have also constructed the variational ground state in terms of free particle basis and performed the Bogoliubov-Valatin transformation to obtain quasi-particle basis which diagonalize the Hamiltonian. We have shown that the wave number $2 \mu$ of the chiral condensate results from the Overhauser instability which generates particle-hole/antiparticle pairs with the total momentum $2 \mu$, or $-2 \mu$.

We have examined the possibility of spatially varying ground state in the Cooper pair model proposed by Chodos, et al. [12 by allowing the Cooper pair condensate to vary periodically in space. By constructing and analyzing the effective potential, we have shown that the ground state with spatially uniform Cooper pair condensate obtained in Ref. [12] gives the true ground state of this model.

We should note that our model, which contains a single chemical potential common to $N$-flavor fermions, does not quite mimic the QCD motivated $(3+1)$-dimensional model in Refs. 88,9,10 in which the mismatch in the Fermi surfaces of up and down quarkes are the essential ingredient for the crystalline color superconducting phase. It would be very interesting to construct a $(1+1)$-dimensional model which possesses the analogous structure and work it out in the similar way as done in this paper. However, the formulation of the large- $N$ field theory with two different chemical potentials is highly nontrivial and we did not enter into the problem in this paper.

The spatial variations of Cooper pair condensate, if it forms, is due to the condensation of 
particle-particle pairs (or antiparticle-antiparticle pairs) with nonvanishing total momenta, as we observe from the Bogoliubov-Valatin transformation. It means that there is nozero expectation value of fermionic current. In other words, the fermionic current becomes an order parameter for the breakdown of the spatial homogeneity.

On the other hand, the role of order parameter is played by fermion number in the chiral Gross-Neveu model, so that the breakdown of the spatial homogeneity is caused explicitly by the chemical potential (in fact, the symmetry is restored at $\mu=0$ ). In the Cooper pair model, however, this symmetry breakdown is caused spontaneously if it occurs. The result, therefore, shows that the spatial homogeneity is not sopntaneously broken in our models.

\section{ACKNOWLEDGEMENTS}

I wish to thank Professor W. A. Bardeen for suggesting possible connection between the regularization scheme employed in this paper and the spectral flow due to chiral rotation in the context of chiral anomaly. I also thank Professor H. Minakata for suggesting the problem and for careful reading of this paper.

\section{APPENDIX A: ULTRAVIOLET REGULARIZATION}

In this Appendix we explain the ultraviolet cutoff procedure given in Eq. (16) in detail.

If we set $|\sigma|=0$ in Eq. (17) in the chiral Gross-Neveu model, it becomes a free field theory. In this case the quasi-particle description should reduce to the free field description. Eq. (10), however, differs from Eq. (22) by the factor $e^{ \pm i K x \gamma^{5}}$. We will show below that the superficial difference between the quasi-particle and the free-particle description is removed by the ultraviolet cutoff prescription.

From the Bogoliubov transformation (23) at $|\sigma|=0$ we first obtain

$$
\begin{array}{ll}
a_{p}=a_{p+K}^{(0)} & \text { for } p>0, \\
a_{p}=a_{p-K}^{(0)} & \text { for } p<0 .
\end{array}
$$

It is natural that the free field description possesses naive momentum cutoff, $|q|<\Lambda / 2$ in Eq. (22). We observe from Eq. (A1) that the cutoff in the $a$-branch becomes $-\Lambda / 2<$

$p-K=q<-K$ and $K<q=p+K<\Lambda / 2$, i.e. $|p|<\Lambda / 2-K$. The remaining mode of free fermion, $-K<q<K$, comes from the $b$-branch:

$$
\begin{array}{ll}
b_{p}=a_{-p+K}^{(0) \dagger} & \text { for } \quad 0<p<K, \\
b_{p}=-a_{-p-K}^{(0) \dagger} & \text { for } \quad-K<p<0 .
\end{array}
$$

Finally, the rest of the Bogoliubov transformation,

$$
\begin{array}{ll}
b_{p}=b_{p-K}^{(0)} & \text { for } \quad p>K, \\
b_{p}=b_{p+K}^{(0)} & \text { for } p<-K,
\end{array}
$$

leads to the cutoff in the $b$-branch, $-\Lambda / 2<p+K=q$ and $q=p-K<\Lambda / 2$, namely $|p|<\Lambda / 2+K$. In addition, we can verify by using Eqs. (A1)-(A3) that the quasi-particle 
decomposition (10) with the cutoff (16) is reduced to the free-particle decomposition (22) with the naive momentum cutoff, $|q|<\Lambda / 2$.

In the above discussion, we have proved that the ultraviolet cutoff must be Eq. (16) for $|\sigma|=0$ at least. We believe that this cutoff is correct one at $|\sigma| \neq 0$ because the cutoff procedure must be insensitive to the vacuum expectation value, as mentioned in Sec. IIT; thus, this cutoff is required to provide the state $|\sigma\rangle$ with the correct fermion number.

Now, we comment on the cutoff prescription from a different point of view. We enclose the system in a finite box of length $L$ for the purpose of counting the number of modes correctly. We shall impose periodic boundary conditions on $\psi_{i}(x)$ and $\psi_{i}^{c}(x)$. This leads to the following conditions on $K$ and $p$ because of the chiral phase factor in wave functions (8),

$$
K=\frac{\pi B}{L}, \quad p=\frac{2 \pi}{L}\left(n+\frac{1}{2} B\right)
$$

where $B$ and $n$ are integer. One can see from the cutoff (16) that $n$ take $\left(2 N_{\Lambda}-B+1\right)$ values from $-N_{\Lambda}$ to $N_{\Lambda}-B$ for $\bar{a}$-branch, and $\left(2 N_{\Lambda}+B+1\right)$ values from $-N_{\Lambda}-B$ to $N_{\Lambda}$ for $\bar{b}$-branch, respectively, for fixed $B$ (i.e., fixed $K$ ). Here, $N_{\Lambda}=\Lambda L / 4 \pi$ is an explicit cutoff parameter. The number of modes included in divergent sums to evaluate the effective potential (14) is kept constant, independently of $B ;\left(2 N_{\Lambda}-B+1\right)+\left(2 N_{\Lambda}+B+1\right)=2\left(2 N_{\Lambda}+1\right)$. We depict the cutoff prescription in Fig. 3. This figure tells us that the cutoff implies summing modes in order from above with respect to the absolute value of energy, keeping the total number of modes fixed when $\mu=0$.

We also note that the fermion number of the state $|\sigma\rangle$ results from the difference of mode between $\bar{a}$ - and $\bar{b}$-branch when we take the cutoff as Fig. 3 . Since $\bar{a}$-branch carries fermion number $-\frac{1}{2}$ and $\bar{b}$-branch carries $+\frac{1}{2}$ as one can find from Eq. (12), the fermion number of $|\sigma\rangle$ becomes $-\frac{1}{2}\left(2 N_{\Lambda}-B+1\right)+\frac{1}{2}\left(2 N_{\Lambda}+B+1\right)=B$ times $N$ of flavor, namely, $N L \frac{K}{\pi}$. It is consistent with the first equation of Eq. (27) because we now ignore the region where $\omega_{-p \bar{a}, \bar{b}}>0$.

For the Cooper pair model, we can discuss the cutoff procedure as the chiral Gross-Neveu model. By comparing Eq. (33) to Eq. (22), we obtain the Bogoliubov transformation

$$
\begin{array}{r}
K<p:\left\{\begin{array}{l}
a_{p}=a_{p+K}^{(0)} \cos \frac{1}{2} \theta_{p_{+}}-a_{-p+K}^{(0) \dagger} \sin \frac{1}{2} \theta_{p_{+},} \\
b_{p}=b_{p-K}^{(0)} \cos \frac{1}{2} \theta_{p_{-}}-b_{-p-K}^{(0) \dagger} \sin \frac{1}{2} \theta_{p_{-}},
\end{array}\right. \\
-K<p<K:\left\{\begin{array}{l}
a_{p}=a_{p+K}^{(0)} \cos \frac{1}{2} \theta_{p_{+}}+b_{p-K}^{(0)} \sin \frac{1}{2} \theta_{p_{+}}, \\
b_{p}=a_{-p+K}^{(0) \dagger} \cos \frac{1}{2} \theta_{p_{-}}-b_{-p-K}^{(0) \dagger} \sin \frac{1}{2} \theta_{p_{-}},
\end{array}\right. \\
p<-K:\left\{\begin{array}{l}
a_{p}=b_{-p-K}^{(0) \dagger} \cos \frac{1}{2} \theta_{p_{+}}+b_{p-K}^{(0)} \sin \frac{1}{2} \theta_{p_{+}}, \\
b_{p}=a_{-p+K}^{(0) \dagger} \cos \frac{1}{2} \theta_{p_{-}}+a_{p+K}^{(0)} \sin \frac{1}{2} \theta_{p_{-}}
\end{array}\right.
\end{array}
$$

The condition in which the quasi-particle description is reduced to the interaction-free description at $|\Delta|=0$ in spite of the presence of $K$, leads to the cutoff (16).

We also depict the same figure as Fig. 3 because the dispersion relations of quasi-particles in the two models coinside with each other at $\mu=0$. In the Cooper pair model, however, the quasi-particle does not carry definite fermion number, but the fermion current. Therefore, the fermion current of the state $|\Delta\rangle$ is generated as we vary the value of $K$, as indicated in Eq. (39). This means from the viewpoint of the Bogoliubov transformation (A5) that 
$|\Delta\rangle$ is composed of two (free)fremions pairing with total momentum $2 K$ or two antifermions pairing with total momentum $-2 K$. 


\section{REFERENCES}

[1] D. Bailin and A. Love, Phys. Rep. 107 (1984) 325.

[2] M. Alford, K. Rajagopal and F. Wilczek, Phys. Lett. B422 (1998) 247; M. Alford, K. Rajagopal and F. Wilczek. Nucl. Phys. B537 (1999) 443; J. Berges and K. Rajagopal, Nucl. Phys. B538 (1999) 215.

[3] D. T. Son, Phys. Rev. D59 (1999) 094019; N. Evans, J. Hormuzdiar, S. D. H. Hsu and M. Schwetz, Nucl. Phys. B581 (2000) 391; for a recent review, see K. Rajagopal and F. Wilczek, hep-ph/0011333.

[4] D. V. Deryagin, D. Yu. Grigoriev and V. A. Rubakov, Int. J. Mod. Phys. A7 (1992) 659.

[5] E. Shuster and D. T. Son, Nucl. Phys. B573 (2000) 434.

[6] B. -Y. Park, M. Rho, A. Wirzba and I. Zahed, Phys. Rev. D62 (2000) 034015.

[7] R. Rapp, E. Shuryak and I. Zahed, Phys. Rev. D63 (2001) 034008.

[8] M. Alford, J. A. Bowers and K. Rajagopal, Phys. Rev. D63 (2001) 074016.

[9] J. A. Bowers, J. Kundu, K. Rajagopal and E. Shuster, Phys. Rev. D64 (2001) 014024.

[10] A. K. Leibovich, K. Rajagopal and E. Shuster, hep-ph/0104073.

[11] D.J. Gross and A. Neveu, Phys. Rev. D10 (1974) 3235; S. Coleman, Aspects of Symmetry (Cambridge Press, 1985).

[12] A. Chodos, H. Minakata and F. Cooper, Phys. Lett. B449 (1999) 260.

[13] A. Chodos, F. Cooper, W. Mao, H. Minakata and A. Singh, Phys. Rev. D61 (2001) 045011.

[14] S. Coleman, Commun. Math. Phys. 31 (1973) 259; N.D. Mermin and H. Wagner, Phys. Rev. Lett. 17 (1966) 1133.

[15] E. Witten, Nucl. Phys. B160 (1979) 57.

[16] V. Schön and M. Thies, hep-th/0008175.

[17] U. Wolff, Phys. Lett B157 (1985) 303; A. Chodos and H. Minakata, Phys. Lett A191 (1994) 39.

[18] J. D. Bjorken and S. D. Drell, Relativistic Quantum field (McGraw-Hill, 1965).

[19] R. Rajaraman, Solitons and Instantons (North-Holland, Amsterdam, 1982); A. Rebhan and P. van Nieuwenhuizen, Nucl. Phys. B508 (1997) 449.

[20] A. W. Overhauser, Phys. Rev. 128 (1962) 1437.

[21] J. Goldstone and F. Wilczek, Phys. Rev. Lett. 47 (1981) 986; R. MacKenzie and F. Wilczek, Phys. Rev. D30 (1984) 2194.

[22] Y. Nambu, Phys. Rev. 117 (1960) 648. 


\section{FIGURES}

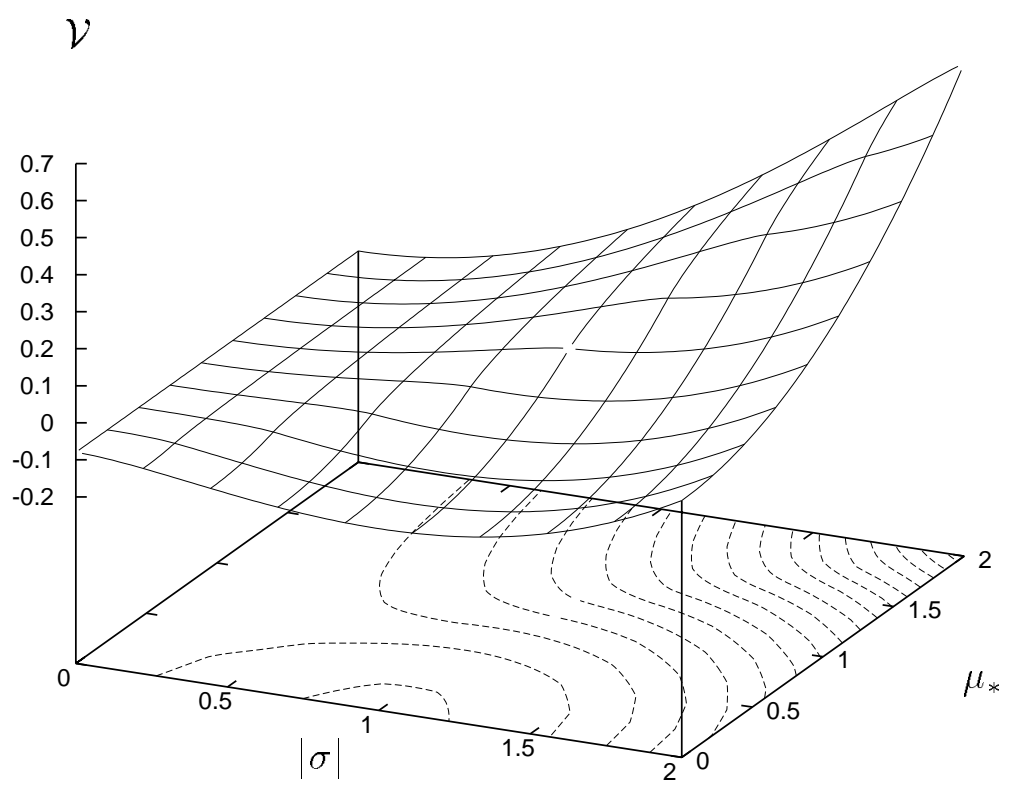

FIG. 1. The effective effective potemtial for the chiral Gross-Neveu model. Each axis is scaled by $m=m_{0} e^{1-\pi / \lambda}$. The contour of the effective potential is depicted on the bottom of $3 \mathrm{D}$ plot. For the Cooper pair model, replace $|\sigma|$ and $\mu_{*}$ by $|\Delta|$ and $K$, respectively.

(a)

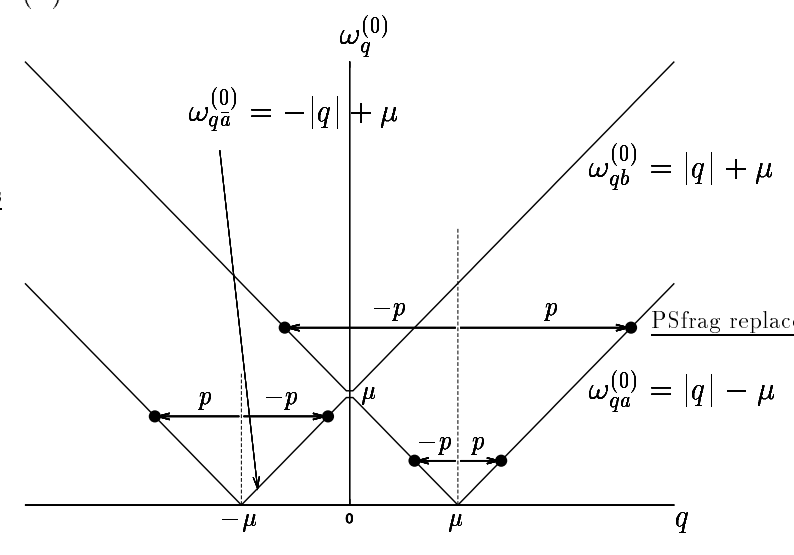

(b)

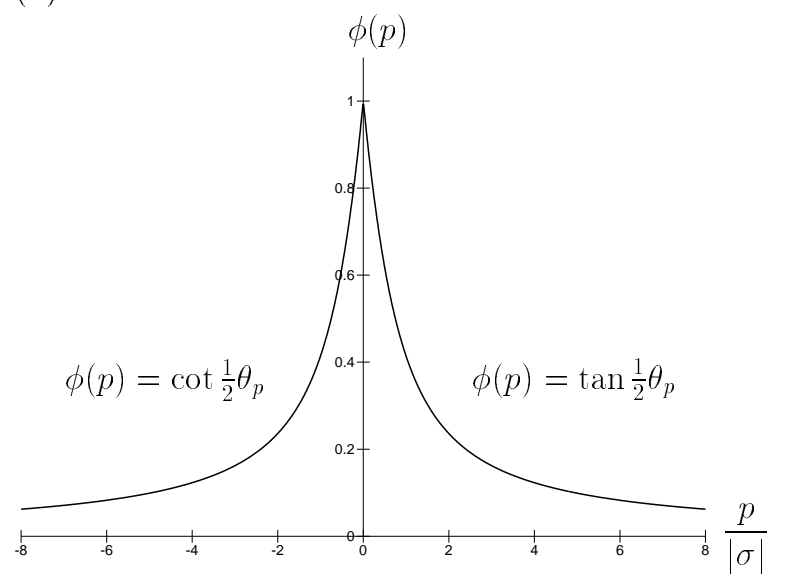

FIG. 2. (a) The manner of pairing for the chiral Gross-Neveu vacuum. $\omega_{q}^{(0)}$ is free particle energy; thus, $\omega_{q a}^{(0)}$ is of the fermion, $\omega_{q \bar{a}}^{(0)}$ is of the hole and $\omega_{q b}^{(0)}$ is of the antifermion. The pairing with total momentum $2 \mu(p>0)$ or $-2 \mu(p<0)$ yields. (b) Pairing wave function $\phi(p)$. 
(a) $B=0(K=0)$

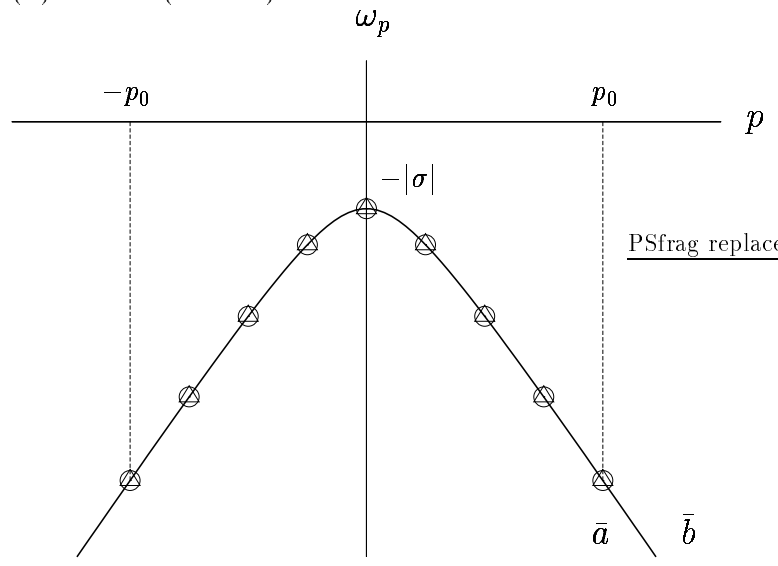

(b) $B=1(K=\pi / L)$

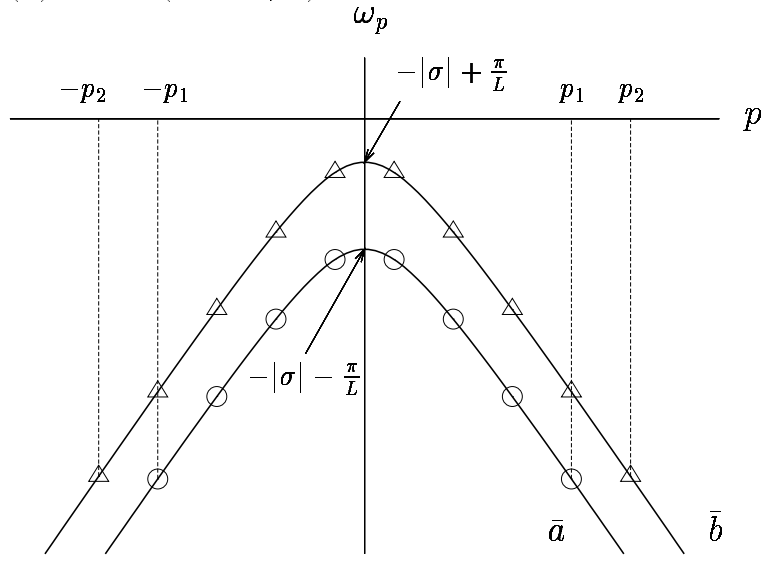

FIG. 3. The cutoff scheme at $\mu=0$. The effective potential is given by the sum of the energies at circles $(\bar{a}$-branch $)$ and triangles $(\bar{b}$-branch $)$. The parameters in the graphs are $p_{0}=\frac{2 \pi}{L} N_{\Lambda}=\frac{\Lambda}{2}$, $p_{1}=\frac{2 \pi}{L}\left(N_{\Lambda}-\frac{1}{2}\right)=\frac{\Lambda}{2}-\frac{\pi}{L}$ and $p_{2}=\frac{2 \pi}{L}\left(N_{\Lambda}+\frac{1}{2}\right)=\frac{\Lambda}{2}+\frac{\pi}{L}$, respectively. 